\title{
Distributed Intelligent Automation Solutions for Self-adaptive Manufacturing Plants
}

\author{
Alessandro Brusaferri, Andrea Ballarino, and Emanuele Carpanzano \\ Institute of Industrial Technologies and Automation, \\ National Research Council, via Bassini 15, \\ 20133 Milan, Italy \\ \{alessandro.brusaferri, andrea.ballarino\} @itia.cnr.it, \\ \{emanuele.carpanzano\}@itia.cnr.it
}

\begin{abstract}
Nowadays, a new generation of responsive factories is needed to face continuous changes in product demand and variety, and to manage complex and variant production processes. To such an aim, self-adaptive automation solutions are required, capable to adapt their control strategy in real-time to cope with planned as well as unforeseen product and process variations. In such a context, the present paper describes an automation solution based on a modular distributed approach for agile factory integration and reconfiguration, integrating a knowledge based cooperation policy providing self-adaptation to endogenous as well as exogenous events. The proposed approach is discussed through its application to a plant for customized shoe manufacturing.
\end{abstract}

Keywords: Distributed Control Systems, Multi-Agent, IEC 61499, Reconfigurable Manufacturing Systems.

\section{Introduction}

To face new consumer centered manufacturing paradigms, like mass customization and personalization, factories must be capable to adapt themselves in real time to continuously changing market demand. Thus, the whole production cycle for small or even single batches has to be executed in very short times, i.e. a few days or even hours. In order to properly approach such complex and strict requirements adaptive knowledge based production systems have to be developed. In particular, the conception and development of a new generation of automation solutions, that integrate all factory levels from machines controls up to shop-floor supervision and production planning in a unique real time framework, is mandatory.

Future factory automation systems have to be modular, open, agile and knowledge based in order to promptly self-adapt themselves to changing exogenous conditions, like consumers expectations, market dynamics, design innovation, new materials and components integration. For such an aim, a new generation of intelligent, highlyinteroperable and self-reconfigurable control systems is a fundamental enabling technology. 
To tackle such a challenge, agile manufacturing paradigms - particularly flexible manufacturing systems (FMS) - have been adopted, often proving to be expensive and difficult to manage due to overall complexity. Furthermore, the integration of flexibility capability is not feasible for any kind of application. Therefore, to overcome such barriers and to provide cost effective flexible solutions, Reconfigurable Manufacturing Systems (RMS) have been introduced, characterized by strongly modular architectures and easy reconfiguration capabilities. In such a direction [1] identify modularity, integrability, diagnosability, customization and convertibility as key features of a RMS.

Among these, system modularity can surely be considered the most important, as outlined in [2] where implications and relationships between the architecture of a logic control system, its modularity and overall level of system reconfigurability, are discussed.

The problem of agile system reconfiguration has been faced mainly from the mechanical point of view with the development of easily pluggable mechatronic solutions. Nevertheless, proper solutions addressing a fully modular and reconfigurable control system have still to be identified.

As a matter of fact, present automation approaches and architectures - adopted in industry - are still based on rigid, loosely-coupled solutions, difficult to manage and to adapt, while current methods and tools for control system programming do not effectively support control system reconfigurability [3].

The integration of a new device within the overall production system or the replacement of a faulty device very often requires a critical stop of the system, to perform physical connections and allocations of the new device, as well as partial/total reprogramming of some parts of the control system, and modifications in production plans, which need time consuming commissioning operations to be executed afterwards [4].

The present paper proposes a self-adaptive control solution in order to support the RMS agility. Particularly, section II briefly analyzes the current state of the art related to the development of self-adaptive control solutions for Reconfigurable Manufacturing Systems and summarizes the main features to be guaranteed; section III presents the proposed overall system architecture; section IV reports conclusions and next developments are acceptable.

\section{Requirements and Available Technologies}

\subsection{RMS Architecture}

Typically, Reconfigurable Manufacturing Systems architectures may be structured into three major hierarchical layers as shown in Figure 1: unit level, cell level and system level. In particular, the overall system is composed by the aggregation of different cell modules, according to the system layout, while, in its turn, a cell module is constituted by the aggregation of more units. Specifically, units can be either operating machines or modules dedicated to parts handling and transportation, e.g. conveyors, rotating tables and manipulators. 
Starting from last sampled status of underlying controlled objects, each module of the RMS control system - being unit control module, cell control module or system control module - decides and performs its control actions according to the fixed decision policy defined and hard-coded during the control system development phase. The interactions between modules are established ex ante and implemented according to fixed bindings between different modules interfaces.
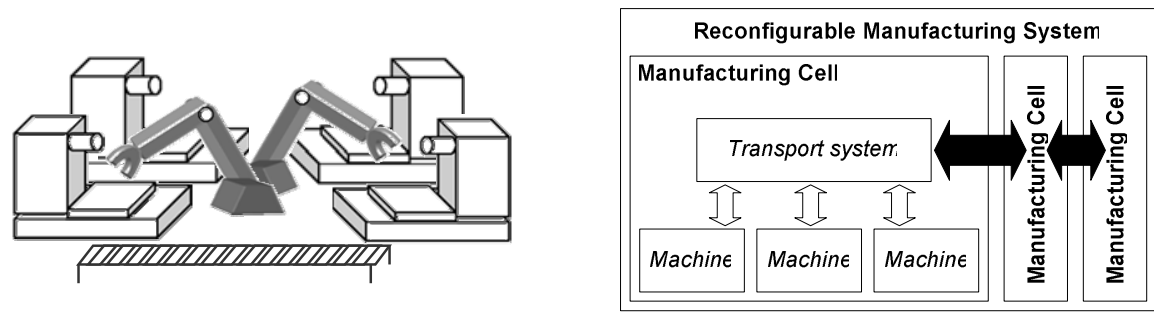

Fig. 1. Reconfigurable Manufacturing System Overall Architecture

Such a strictly coupled architecture is very difficult to modify during RMS reconfiguration phases, as time and costs required for such operations grow rapidly with the increase in system complexity. To such an aim, a new generation of loosely -coupled control architectures, based on a distributed easy-reconfigurable architecture, integrating a flexible knowledge based decision policy, has to be introduced.

\subsection{Currently Available Enabling Technologies}

Today, the required levels of modularity and distribution of control solutions are not properly adopted in industrial practice due to the lack of well defined and accepted reference models. Major consequences of this lack are twofold. First of all, implemented control and supervision strategies are today typically based on rigid centralized approaches organized into strictly coupled sequences of operations. Difficulties in reconfiguration and in real-time adaptation to production needs are most relevant resulting problems. As a second major consequence, suitable readability, portability and integrability of overall control and automation solutions are not supported. Therefore, the capitalization and reuse of company specific know-how on process and control is very difficult. Flexibility, optimization and failure management features are not properly tackled as well, thus critically impacting the overall production process efficiency and fault tolerance.

Great research efforts have been spent in recent years to conceive a common and well accepted reference model. In such a direction, the multi-agent system (MAS) paradigm shall be mentioned as one the major efforts for development of robust distributed control systems. Such a paradigm is based on autonomous modules, which integrate knowledge-base for decision making inference, high-level communication protocols and languages to support loosely coupled architectural organizations [5]. Despite its potential capabilities, major limits of such an approach for complex industrial test-cases reside in difficulties of guaranteeing strict execution time requirements. 
Recently different research projects as SIRENA ${ }^{1}$, RIMACS $^{2}$, SOCRADES $^{3}$, have considered an alternative architectural solution for developing systems composed of autonomous and interoperable units: the Service Oriented Architecture - SOA. Such paradigm is characterized by coarse-grained service interfaces, loose coupling between service providers and service consumers, and message-based, asynchronous communication [6]. Leveraging the SOA paradigm allows for services to be re-used across processes and systems, and systems to be "built for change". Reliability is improved as applications and systems can be made up of tested and proven components. SOA offers the potential to provide the necessary system-wide visibility and interoperability in complex systems subject to frequent changes and operating in a multi-vendor environment. Nonetheless, Service Oriented solutions are still unable to reach the hard-real time constraints in particular for controlling complex manufacturing processes with huge amounts of data and high numbers of units. Furthermore, the decisional logic is not supported by SOA, thus, intelligence has to be integrated onto the SOA level.

In particular, self-adaptivity needs self-interoperability of information: the knowledge has to be structured in order to be understood by autonomous intelligent agents able to interpret the domain conditions and taking the proper decisions. For such reason, a major research effort is ongoing widespread to exploit the adoption of Semantic Web approaches into the factory automation domain. Such paradigm is oriented to the implementation of machine interpretable information supporting the implementation of intelligent control solutions based on formal knowledge models. The formal definition of classes properties and instances allows inferring new knowledge from knowledge already structured into a model.

To cope with real time distributed control, a formal model has been proposed within the IEC 61499 standard of the International Electrotechnical Commission, promoted by the international O3NEIDA network ${ }^{4}$. The normative states the common interfaces and structure of the embedded solutions from simple basic function blocks, to composite functional integrations, up to overall control systems applications. It also provides guidelines for the application distribution within multi-vendor control execution devices. Nonetheless, it does not provide structured indications related to selfadaptive control systems design.

Several research actions have been also oriented to the integration of the low level, hard real-time, control layer and the high level, low real-time, control/supervision layer. In particular, [7] propose an interface for the integration of an heterogeneous low level control based on IEC 61499 standard and a Multi-Agent System for the manufacturing domain. [8] propose the integration of Service Oriented Architecture and a Multi-Agent System (MAS) in order to build a control architecture suitable for automated reconfigurability. [9] introduce a holonic manufacturing control architecture integrated with the logic control layer, designed to improve the agility and reconfigurability of production systems.

\footnotetext{
${ }^{1}$ SIRENA Project www.sirena-itea.org.

${ }^{2}$ RIMACS Project www.rimacs.org.

${ }^{3}$ SOCRADES Project www.socrades.eu.

${ }^{4}$ O3NEIDA Network www.oooneida.org.
} 
Despite the performed research effort and the emerged benefits, such paradigms are currently not implemented within industrial solutions. In fact, the real world applicability needs to be demonstrated through complex industrial test cases highlighting the concrete advantages and providing guidelines for industrial applications.

\section{Deployed Control Architecture}

Present section describes main architectural and functional aspects regarding the proposed self-adaptive control solution. In particular, a real industrial plant is considered as a test-case in order to properly support the description and provide application details. Nonetheless, proposed solutions can be integrated within any manufacturing automation system. Obviously, a proper configuration of specific control rules and knowledge base classes is required.

\subsection{The Focused Industrial Plant}

Before starting the description of the deployed control architecture, the considered real industrial application, an innovative shoe manufacturing plant managed by ITIACNR, is presented, see Figure 2. For the sake of brevity, a simplified version of a part of the manufacturing system is here considered.
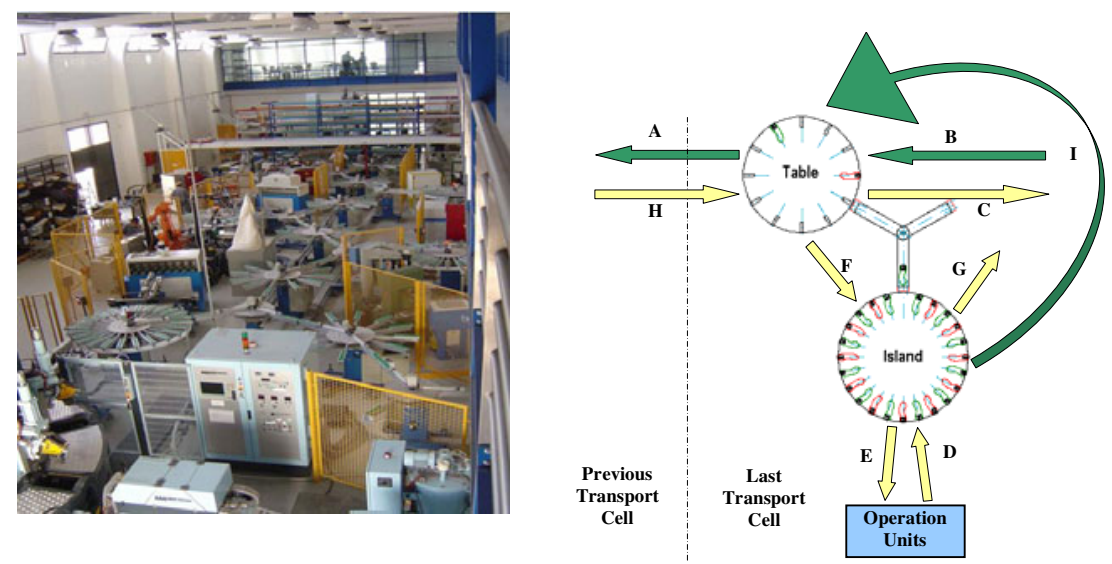

Fig. 2. Shoe manufacturing plant and work pieces flows

The considered manufacturing system integrates an innovative transport line for moving the semi-finished shoes from a machining station to another one according to operations requests. The innovative molecular structure enhances the modularity, scalability, integrability and reconfigurability properties of the production system, increasing the overall flexibility of the plant.

The basic element of the molecular structure is the "Tern", which is constituted by two rotating tables, called "Table" and "Island", and by a rotating three arms manipulator. The Table moves the semi-finished shoes either to the next Tern or to the Island 
of the same Tern. Moreover, it moves backward the lasts flowing back towards the warehouse (the last is the object around which the semi-finished shoe is built upon). The Island directs the semi-finished shoes towards the different machining stations, laid around the Island itself. The manipulator carries out the transport of the semifinished shoes and lasts between Tables and Islands. The arrows in Figure 2 depict the possible movements that involve a generic semi-finished shoe or last in a generic single Tern.

\subsection{The Deployed Control Solution}

Starting from the process specification, a modular and distributed control architecture has been defined, integrating a real-time IEC 61499 distributed control layer and a multi-agent semantic enriched control and supervision layer. First of all, to achieve the desired agility objectives, object-oriented concepts have been exploited within control system development. For such an aim, the IEC 61499 standard has been adopted as design paradigm due to its orientation to the deployment of modular and distributed control solutions [10]. In particular, the molecular line has been considered as a set of interacting Terns, each one with its own independent control system. Each Tern control module communicates with the related Table, Island and Manipulator control modules, as shown in Figure 3. Moreover, each Tern control module is connected to the adjacent Terns control system modules, to coordinate the exchanges of semi-finished shoes and lasts.

Furthermore, each basic function block encapsulates the control logic by means of a state machine responsible for the activation of dedicated IEC $61131^{5}$ based control algorithms depending on run-time events and conditions. For more details regarding function blocks internal structure see [3].

Tern Control modules represent the low level control layer, responsible for real time control tasks. In particular, tasks dedicated to manage nominal and failure operating condition have been integrated.
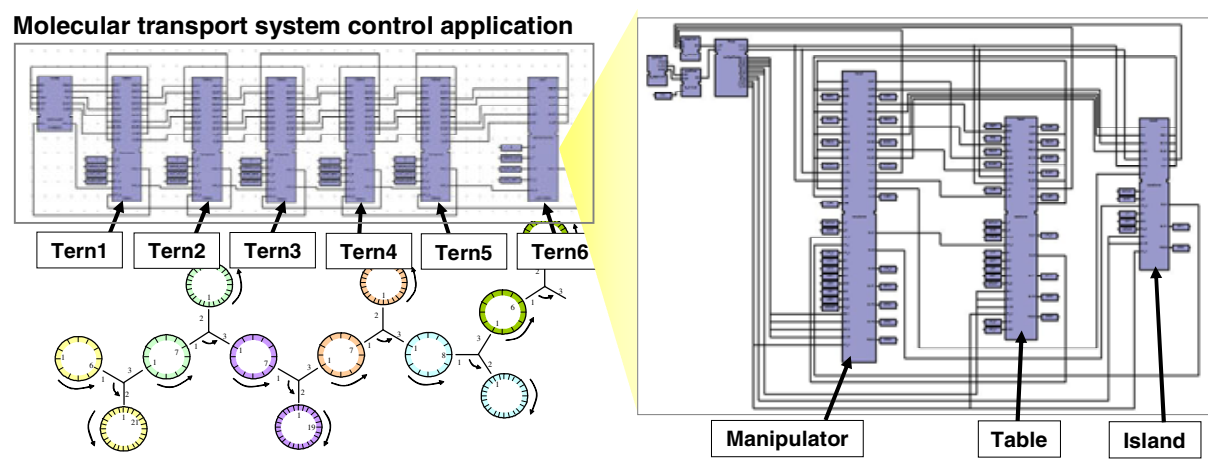

Fig. 3. Control architecture of the Molecular transport line

\footnotetext{
${ }^{5}$ International Standard IEC 61131 - www.iec.ch.
} 
Such control modules have been implemented within the ISaGRAF 5 Workbench ${ }^{6}$. By supporting IEC 61499, such environment acts as backbone for the overall application development, from design to implementation and validation. Furthermore, the distributed hardware architecture can be defined by properly assigning the Resources (Virtual PLCs) executed within each device. Moreover, each device integrates I/O interfaces connected to the factory field.

The application can then be designed as a unique control program. Furthermore, the developed control application can be generated into target-independent $\mathrm{C}$ code, executable by a firmware, which emulates a virtual machine on hardware, being it an Industrial PC, an embedded board or a tiny controller. Therefore, portability and scalability of the developed application is guaranteed.

In order to provide self-adaptive capabilities to the deployed automation solution while reconfiguring manufacturing resources (i.e. adding, removing operational units or integrating manufacturing functions) a multi-agent control and supervision layer has been integrated onto the object-oriented real-time control layer. In particular, each Tern control module has been connected to a Tern agent, responsible for the interaction within the high level control layer. The interface between the IEC 61499 modules and the multi-agent layer, deployed in Java language, has been developed by means of the Java Native Interface provided by Sun Microsystems Inc ${ }^{7}$.

Besides, the higher level control layer is responsible for the coordination of the low level real-time control layer in response of predicted as well as unforeseen events. In fact, the decisional logic has to be dynamically adapted to follow changing products features/requirements contextually to machine operational capabilities reconfiguration, tools integrations, and new machines implementation into production cells. The higher level control layer is also aimed at interacting within MES and ERP systems so to receive incoming production orders as well as provide a run-time updated factory image to the management layers. To such an aim, dedicated interaction mechanisms have been implemented.

Furthermore, the self-adaptation mechanism has also to react when operational capabilities become unavailable due for example to failures or maintenance operations. To such an aim, a dedicated management agent has been integrated, implementing a knowledge based reasoner connected to complementary knowledge model based on a common formal domain description:

- Product knowledge base model: aimed at the structuring of information related to products to be processed by the manufacturing system, as required operations and priorities with reference to the overall production plan.

- Resource knowledge base model: aimed at the formal description of the intelligent units run-time integrated within the production facility, i.e. the operations provided and execution state.

Such models are dynamically updated by means of a dedicated Interface agent, in order to be aligned to the process evolution, supporting the optimal real-time decision strategy. Two dedicated model update mechanisms have been implemented: the first has in charge the management of the products entering/leaving the system

\footnotetext{
${ }^{6}$ Isagraf Workbench - www.isagraf.com

${ }^{7}$ Java Native Interface - http://java.sun.com/j2se/1.4.2/docs/guide/jni/
} 
and their operational state change due to processed manufacturing tasks performed within the RMS; the second supervises the resources integrated within the system, updating their available operations depending on their evolving execution state and/or reconfigurations.

Thanks to the adoption of such an asynchronous policy, the model adaptation task is called only when required, reducing the overall execution time. Furthermore, semantically-rich based descriptions have been adopted to implement the knowledge based solution. In particular RDF and OWL-DL W3C ${ }^{8}$ standards coming from the Semantic Web area have been considered. Therefore, machine reasoning has been used to perform automatic matchmaking of required and offered services using logical inference, rather than performing hard-coded one-to-one mappings.

Such type of matchmaking enables the use of services that did not exist or were not known when the requestor side was programmed, enabling automation system reconfiguration without control logic reprogramming. The overall control architecture is outlined in Figure 4.
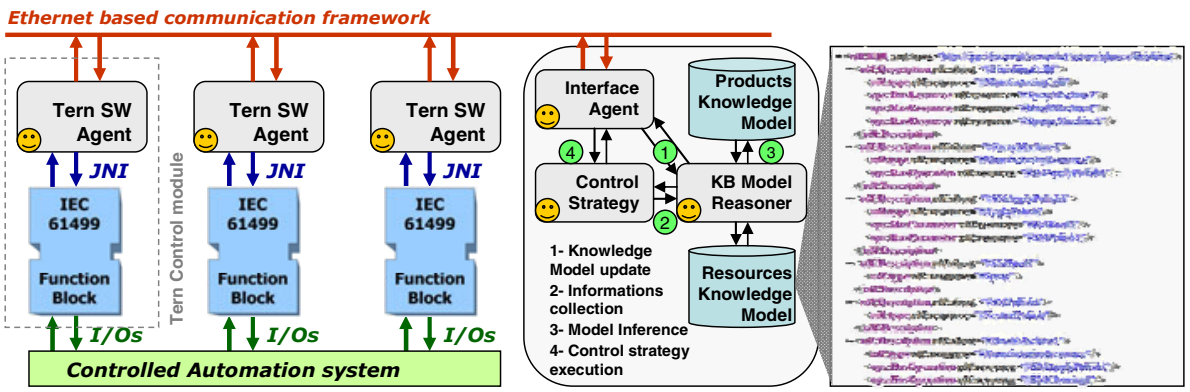

Fig. 4. Overall multi-agent control architecture

Once a new operational unit is plugged into the production system its agent asks for the registration service to the manager passing is description as argument, formally reporting the operations to be provided and the required resources (i.e. tools) to be used during operation execution. The operations to be provided and the resources to be used are described with reference to a common ontology.

The manager interprets the ontological description of the intelligent unit and integrates it into a dedicated knowledge base within the manager architecture. A searching algorithm is then executed in order to find the run-time available manufacturing resources capable of providing the requested operations.

\section{Conclusions}

The present paper describes a self-adaptive control architecture deployed for a real industrial plant. To such an aim, a modular and distributed approach has been adopted, integrating an IEC 61499 based control solution for real-time control

\footnotetext{
${ }^{8}$ OWL Web Ontology Language - http://www.w3.org/TR/owl-guide/
} 
purposes and a semantically enriched multi-agent control layer for dynamic supervision strategy. Main focus of the paper is kept on the description of the overall solution structure, highlighting the capabilities of run-time updating of the knowledge model used for control decisions, while factory products and resources changes occur.

Next efforts will mainly regard the integration of dedicated supervision and on-line dispatching/scheduling facilities onto the deployed control architecture.

\section{References}

1. Koren, Y., Heisel, U., Jovane, F., Moriwaki, T., Pritschow, G., Ulsoy, G., Bruseel, H.V.: Reconfigurable manufacturing systems. Ann. CIRP 1999 48(2), 527-540 (1999)

2. Almeida, E., Luntz, J., Tilbury, D.: Event-Condition-Action Systems for Reconfigurable Logic Control. IEE Transaction on Automation Science and Engineering 4(2), 167-181 (2007)

3. Brusaferri, A., Ballarino, A., Carpanzano, E.: Enabling Agile Manufacturing through Reconfigurable Control Solutions. In: Proc. at 14th IEEE International Conference on Enabling Technologies and Factory Automation (ETFA 2009), Mallorca, Spain (September 2009)

4. Carpanzano, E., Jovane, F.: Advanced Automation Solutions for Future Adaptive Factories. Annals of the CIRP 56(1), 435-438 (2007)

5. Pechoucek, M., Marík, V.: Industrial deployment of multi-agent technologies: review and selected case studies. Autonomous Agents and Multi-Agent Systems Journal, 397-431 (May 14, 2008)

6. Lastra, J., Delamer, I.: Web Services in Factory Automation: Fundamental Insights and Research Roadmap. IEEE Transactions on Industrial Informatics 2(1), 1-11 (2006)

7. Lepuschitz, W., Vallée, M., Merdan, M., Vrba, P., Resch, J.: Integration of a Heterogeneous Low Level Control in a Multi-Agent System for the Manufacturing Domain. In: Proc. at 14th IEEE International Conference on Enabling Technologies and Factory Automation (ETFA 2009), Mallorca, Spain (September 2009)

8. Herrera, V., Bepperling, A., Lobov, A., Smit, H., Colombo, A.W., Lastra, J.L.: Integration of Multi-Agent Systems and Service-Oriented Architecture for Industrial Automation. In: Proc. at IEEE International Conference on Industrial Informatics (INDIN 2008), Daejeon, Korea, July 13-16 (2008)

9. Leitao, P., Restivo, F.: Implementation of a Holonic Control System in a Flexible Manufacturing System. IEEE Transactions on Systems on, Man, and Cybernetics - Part C: Applications and Reviews 38(5), 699-709 (2008)

10. International Electro-technical Commission (IEC), International Standard IEC61499, Function Blocks, part 1-4, IEC, Edition 1.0. (January 2005), http : / /www . iec. ch/ 